\title{
High Performance Computing Tools for Cross Correlation of Multi-Dimensional Data Sets Across Instrument Platforms
}

\author{
Alex Belianinov, ${ }^{1,2}$ Danka Gobeljic, ${ }^{3}$ Vladimir Shvartsman, ${ }^{3}$ Erik Endeve, ${ }^{1,4}$ Eric J. Lingerfelt, ${ }^{1,4}$ \\ Richard Archibald, ${ }^{1,4}$ Sergei Kalinin, ${ }^{1,2}$ Stephen Jesse ${ }^{1,2}$ \\ 1. Institute for Functional Imaging of Materials, Oak Ridge National Laboratory, Oak Ridge, TN, USA \\ 2. The Center for Nanophase Materials Sciences, Oak Ridge National Laboratory, Oak Ridge, TN, USA \\ 3. Institute for Material Science and Center for Nanointegration Duisburg-Essen (CENIDE), University \\ of Duisburg-Essen, Essen, Germany \\ 4. Computer Science and Mathematics Division, Oak Ridge National Laboratory, Oak Ridge, TN, USA
}

Measurements of a single sample system performed across a variety of instruments, at different spatial and energy scales, probing different properties, provide valuable additional information on mechanisms and properties of interest. Typically, since measurements are performed at different areas of the sample, the addition to the overall body of knowledge increases in a disjointed fashion. Overall, the amount of information is enhanced, but the new data does not necessarily correlate all measurements directly. In some cases, it is possible to make multiple measurements at the same site, with natural markers, nanofabricated features or measured responses. However, in these cases the same problems of scale and energy resolution, along with new problems of imaged area registration and data correlation limit the usability of the obtained results, and discourage scientist from pursuing high veracity multiple platform data sets.

In this work we demonstrate techniques used to correlate 2, 3 and 4D data sets that were obtained from the same region, at different resolutions using entirely different instrumentation.[1] Cases of data coming from integrated instruments with multiple probes, as well as physically separated machines, and their respective challenges will be shown and discussed, as shown in Figure 1. Fig. 1 illustrates coregistration of probe microscopy and electron microscopy originated data. Multidisciplinary examples include atomically resolved ultra-high vacuum imaging and spectroscopy for exploring atomic level spatial variability of electronic structure in Fe-based superconductor $\mathrm{Fe}_{1.05} \mathrm{Te}_{0.75} \mathrm{Se}_{0.25}$; [2] as well as a blend of ambient, probe and diffraction techniques made at mesoscopic length scales for studying nanoscale heterogeneity of the polarization reversal in huge strain lead-free relaxor-ferroelectric ceramic/ceramic composites will be presented and discussed.[3,4]

References:

[1] O S Ovchinnikova et al, ACS Nano, 9(4) (2015), p. 4260.

[2] A Belianinov et al, APL Materials, 2(12) (2014), p. 120701.

[3] D Gobeljic et al, Nanoscale, 8(4) (2016), p. 2168.

[4] Research for all authors was supported by the US Department of Energy, Basic Energy Sciences, Materials Sciences and Engineering Division. This research was conducted at the Center for Nanophase Materials Sciences, which is sponsored at Oak Ridge National Laboratory by the Scientific User Facilities Division, Office of Basic Energy Sciences, U. S. Department of Energy. 

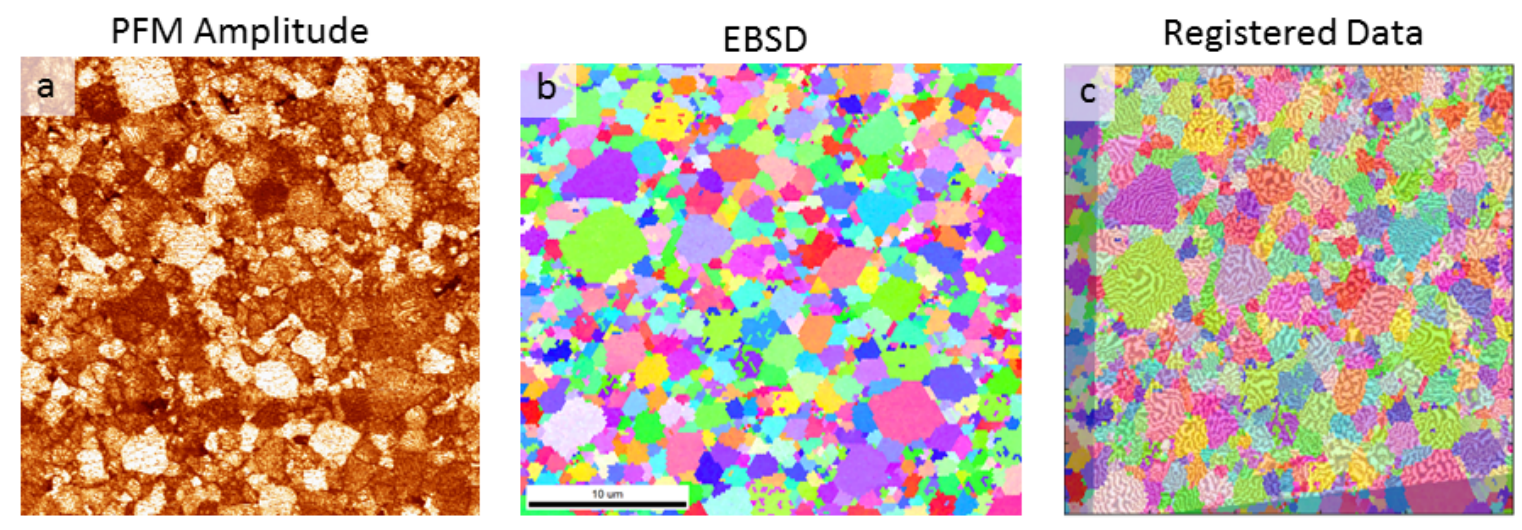

Figure 1. Data obtained from two different instruments in the same region, to be registered. (a) Piezoresponse Force Microscopy (PFM) Amplitude of 0.92BNT-0.06BT-0.02KNN/0.93BNT-0.07BT (ergodic/non-ergodic relaxor) composite collected in an Atomic Force Microscope (AFM), (b) Electron Backscatter Diffraction (EBSD) data from a Scanning Electron Microscope, (c) Two data pieces in (a, b) co-registered onto a single image axis. 